\section{PSYCAUSE}

Revue scientifique étudiante de

l'École de psychologie de I'Université Laval

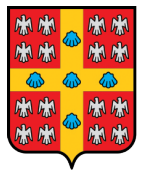

UNIVERSITÉ LAVAL

Faculté des sciences sociales École de psychologie

SEPTEMBRE 2018 - VOL. $8 \mathrm{~N}^{\circ} 2$

\title{
LETTRE OUVERTE SUR L'IMPORTANCE DU FÉMINISME DANS L'ÉCRITURE SCIENTIFIQUE
}

Les membres du comité éditorial de la revue Psycause ${ }^{1, *}$

1 École de psychologie, Université Laval, Québec, Canada

2 revuepsycause@psy.ulaval.ca

\section{Pour citer l'article}

Les membres du comité éditorial de la revue Psycause. (2018). Lettre ouverte sur l'importance du féminisme dans l'écriture scientifique. Psycause: Revue scientifique étudiante de l'École de psychologie de l'Université Laval, 8(2), 4-7. 


\section{LETTRE OUVERTE SUR L'IMPORTANCE DU FÉMINISME DANS L'ÉCRITURE SCIENTIFIQUE}

\section{Les données des participantes aux études en psychologie sont systématiquement exclues des études publiées.}

Du moins, c'est ce que certaines naïves gens ${ }^{1}$ pourraient penser lorsqu'elles lisent des articles, affiches ou résumés d'études en psychologie. Effectivement, il n'est pas rare de lire dans les articles ou autres écrits scientifiques les lignes suivantes: "les participants prennent part à une tâche...» ou «les participants remplissent le questionnaire...» ou même «les résultats des participants suggèrent que...». Pourtant, les sujets qui participent aux expériences dans les laboratoires de l'École de psychologie sont bien souvent des participantes, des femmes; ces dernières sont d'ailleurs majoritaires au sein de notre département! Pourquoi ne sontelles donc pas identifiées comme telles?

Il s'agit évidemment ici d'une question de grammaire d'usage, de règles de langues. Nous avons déjà toutes et tous entendu dans nos cours de français que «le masculin l'emporte sur le féminin » ou, tel que maintenant proposé par l'Office québécois de la langue française (OQLF), que «l'accord doit se faire au genre masculin $»^{2}$. Les règles n'ont donc pas changé. Cependant, I'OQLF stipule ceci: «Toutefois, [l'emploi d'appellations au féminin] est recommandé par l'Office québécois de la langue française pour désigner les femmes. Cet emploi est souhaité et encouragé pour représenter les femmes dans les textes et, par là même, rendre visible leur place dans la société ». L'ensemble de la communauté scientifique s'entend pour dire que les textes scientifiques doivent être concis et directs, mais l'ajout des mots «et participantes» affecterait-il réellement la clarté et la concision d'un article, d'une affiche ou d'une thèse?

L'inclusion du féminin dans les textes gagne peu à peu du terrain. À cet effet, l'utilisation récente du terme autrice pour définir une femme qui est à l'origine d'une œuvre a récemment fait surface. Selon l'OQLF, au Québec, le féminin du mot auteur est auteure. Pourquoi alors, autrice? La raison est toute simple: il s'agit en réalité de la première forme féminisée du mot auteur. Les mots auteur et autrice proviennent respectivement des racines latines auctor et auctrix, dont le second est la forme féminine du premier. Selon Evain (2008), le terme autrice était courant du côté de l'usage, dans la langue parlée, et même dans la langue écrite jusqu'à la période de la Renaissance où son utilisation a peu à peu disparu. Les racines latines de ces termes sont également croisées avec celles des mots acteur et actrices (respectivement actor et actrix), couramment utilisés de nos jours. Le terme actor n'a longtemps pas admis de forme féminine, puisqu'il désignait-outre l'homme qui joue la comédie-des fonctions sociales ou des métiers liés au droit, à la justice, à la gestion et à la littérature; bref, des statuts d'intellectuel. Cependant, lorsque le terme acteur est devenu limité à celui qui joue la comédie, qui utilise son corps sans nécessairement utiliser son intellect, le mot actrice a fait son apparition au sein des dictionnaires de langues officiels. L'opposé s'est produit pour le mot autrice lorsque la fonction «auteur» s'est institutionnalisée et qu'un prestige social et littéraire lui fut associée.

\footnotetext{
1 Le mot gens, lorsqu'il est accordé au pluriel, change de genre et devient féminin. Tel qu'indiqué par l'Office québécois de la langue française : «Curieusement, bien que ce nom soit généralement masculin, on le considère parfois comme un nom féminin. [...] De nos jours, lorsqu'un adjectif épithète précède immédiatement le mot gens, on l'accorde au féminin si la forme féminine de cet adjectif diffère de sa forme masculine " (voir la page suivante: http://bdl.oglf.gouv.qc.ca/bdl/gabarit_bdl.asp?id=3428).

2 Voir la page suivante: http://bdl.oqlf.gouv.qc.ca/bdl/gabarit_bdl.asp?id=4015
} 
Petit à petit, sous les recommandations des membres ou des alliés de l'Académie française, créée par la politique culturelle du Cardinal de Richelieu et composée uniquement d'hommes (surprise!), I'utilisation du terme autrice s'est raréfiée, puis disparut. Présenté de façon plus frappante, c'était une façon «de dire aux femmes qu'elles n'avaient pas de légitimité dans [les professions d'intellectuels]» (Alwett, 2016). Même s'il a récemment été féminisé dans sa forme auteure, il n'en demeure pas moins que, à l'oral, aucune distinction ne soit perceptible, ce qui est toujours cohérent avec les objectifs de l'abolition du terme autrice. La juste représentativité des femmes devrait pouvoir être décelable aussi dans le langage courant, et ces mots nous permettant d'apprécier qu'un travail d'érudition puisse être produit par une femme sont nécessaires.

En contrepartie, certains pourraient affirmer: «Mais ce n'est pas officiellement accepté par l'OQLF! » ou «Ça sonne faux à mes oreilles... ». Bien que ces arguments soient recevables, il appert de rappeler l'existence d'un débat qui a émergé au Québec dans les années 1990 à propos de l'utilisation du mot écrivaine. Dans cette décennie, au Québec, le terme écrivaine n'était pas d'usage et on disait plutôt d'une femme qu'elle était écrivain. Le terme a fait son apparition à la suite des pressions de groupes féministes qui revendiquaient la féminisation de ce mot, et de bien d'autres encore. Sur ce sujet, Pierre de Bellefeuille, professeur de français langue seconde à Abbotsford en ColombieBritannique, a écrit ceci dans un article publié dans Le Devoir le 29 mars 1993 :

Depuis quelques années, nous assistons, au Québec et au Canada, à la radio, la télé, et dans les journaux francophones, à la féminisation outrancière de noms tels que médecin, écrivain, député, ministre, gouverneur, docteur, chef, etc. dont la grammaire française a toujours exigé l'emploi au masculin, et que les plus récentes éditions de tous les dictionnaires continuent de définir comme noms masculins. Pour les mettre au féminin, nous a-t-elle toujours enseigné, il suffit de leur ajouter le mot «femme», et le tour est joué: une femme médecin, une femme écrivain, etc. Aurait-on subitement aboli la grammaire? Qu'en est-il exactement?

Autres temps, autres mœurs, heureusement pour nous et pour les «hommes professeures» de français langue seconde! Dans le même article, on peut également lire ceci :

Au Canada même, madame Anne Hébert,
femme de lettres éminente, lors d'une
émission de Littérature au pluriel au
réseau français de Radio-Canada, il
y a quelques années, manifestait son
agacement devant ces féminisations
toutes plus farfelues et saugrenues les
unes que les autres en avouant sans
ambages en avoir ras le bol de se faire
appeler "écrivaine» ou «autrice».

Écrivaine et autrice? Intéressant... Si en à peine 20 ans ce mot a pu prendre sa pleine place et permettre d'écrire-et de dire-haut et fort qu'une écrivaine est une femme qui écrit, il est difficile de croire que le mot autrice ne pourrait pas suivre le même parcours aujourd'hui.

Ayant fait la démonstration que l'inclusion des femmes dans l'écriture de textes est recommandée et que l'utilisation de termes spécifiques au genre féminin est faisable, il peut être important de se questionner sur leur importance. Pourquoi ce débat, et pourquoi cette lettre? Les étudiantes et étudiants ayant suivi le cours Processus cognitifs (PSY-1007) se rappelleront sans doute les études qui montrent que le langage a une incidence sur les représentations mentales. Par exemple, l'étude d'Athanasopoulos (2009) compare la perception de la couleur bleue d'un échantillon de sujets dont la langue maternelle est le grec (pour qui la couleur bleue est nommée par deux mots différents selon que le bleu soit pâle ou foncé) et d'autres 
dont la langue maternelle est l'anglais (pour qui un seul mot est utilisé pour décrire la couleur bleue). La couleur verte, pour laquelle les deux langues possèdent un seul mot afin de la nommer, est aussi utilisée à titre de condition contrôle. À l'aide de la vMMN, un indice électrophysiologique de perception de stimuli rares, les résultats permettent d'observer que la présentation inattendue d'une teinte foncée de bleu parmi plusieurs présentations séquentielles d'une teinte de bleu pâle (et vice-versa) produit une vMMN supérieure chez les sujets grecs qui utilisent deux mots pour désigner ces teintes, que chez les sujets anglophones qui possèdent un seul mot générique pour la couleur bleue. Au contraire, l'impact du changement de teintes de vert sur la vMMN est similaire tant chez les sujets grecs qu'anglais. Il semble donc que le langage influencerait la perception, notamment pour les couleurs. Boroditsky (2017), experte en psychologie cognitive du langage, présente également dans un TED talk très intéressant et pertinent quelques manières par lesquelles le langage affecte notre perception dans différents domaines, notamment dans notre façon de décrire les objets auxquels nous associons des genres (p. ex., le mot pont, féminin en allemand, principalement décrit par des termes comme beau et élégant, associés au genre féminin; et masculin en espagnol, et principalement décrit par des termes comme fort et long, associés typiquement au genre masculin).

II semble donc que le langage ait un impact sur la perception de notre monde, et ce, sans nécessairement que l'on ne s'en rende compte. Alors, pourquoi ce débat, et pourquoi cette lettre? Pour changer nos perceptions, pour rappeler à notre inconscient collectif que les femmes ont leur place en tant qu'autrices d'articles scientifiques, que professeures et chercheuses universitaires, et que cet intérêt envers la science peut commencer dès qu'elles deviennent participantes pour la première fois à une étude en psychologie. La place des femmes dans la science reste encore à faire. Une étude de Stoet et Geary (2018) montre que la différence entre les genres en regard des forces et de la poursuite aux études dans les domaines des sciences, technologies, ingénieries et mathématiques augmente paradoxalement selon que les valeurs d'égalité des genres soient ancrées au sein d'une nation. Selon Statistiques Canada, les femmes canadiennes occupaient, en 2011, 22,7\% des emplois au sein de professions scientifiques de niveau universitaire alors que les hommes occupaient plutôt 77,3\% de ces emplois (Dionne-Simard, Galarneau \& LaRochelle-Côté, 2016). Faisant partie d'un domaine d'étude où les femmes occupent une grande place et dont les valeurs d'égalité, d'intégrité, de rigueur et de respect sont primordiales, il est de notre devoir d'agir concrètement afin que les femmes soient reconnues à leur juste valeur et à l'égal des hommes. L'utilisation d'un langage spécifique à cette fin est une façon simple d'agir et elle s'avère porteuse d'un message plus grand-pour la société et pour nos propres perceptionsqui rappelle que les femmes font aussi partie intégrante de la science.

Contribuer à ce changement d'habitude et, ultimement, de mentalité est le travail de chacune et chacun d'entre nous. Conséquemment, la revue Psycause se positionne en tant que revue féministe et incite les membres de l'École de psychologie à faire de même en adaptant leur langage écrit et oral en ce sens. Dès lors, la revue Psycause encourage les autrices et auteurs d'articles scientifiques à utiliser un langage épicène (c.-à-d. neutre de genre), tel que promu par l'Université Laval, afin d'inclure l'ensemble des membres de la communauté sans égard au genre, qu'il soit féminin, masculin ou non-binaire. Dans des situations où un tel langage est difficile, répétitif ou qu'il alourdit réellement le texte, la revue Psycause suggère de privilégier l'égalité dans la visibilité des femmes et des hommes en intégrant du mieux possible les deux genres (par l'utilisation 
de doublets complets; p. ex., participantes et participants) ou en adoptant un langage permettant de démarquer la présence de femmes ou d'hommes en sciences et dans la société (en faisant référence à une chercheuse ou une autrice lorsque la personne concernée est une femme et en utilisant les termes chercheur et auteur lorsqu'il s'agit plutôt d'un homme).

Chercheuses et chercheurs, étudiantes et étudiants, et professeures et professeurs, nous espérons que ce message ait une incidence sur votre façon d'écrire et sommes impatientes et impatients de voir la différence qu'un tel geste puisse avoir sur les membres de la communauté scientifique.

Les membres du comité éditorial de la revue Psycause $^{3}$

\section{Références}

Alwett, A. (2016, 22 février). Auteur, auteure ou autrice? Récupérée sur http://www. audreyalwett.com/auteur-auteure-ou-autrice/

Athanasopoulos, P. (2009). Cognitive representation of colour in bilinguals: The case of Greek blues. Bilingualism: Language and Cognition, 12,83-95. doi:10.1017/S136672890800388X

Boroditsky, L. (2017). How language shapes the way we think. Présentation orale effectuée aux TEDWomen 2017, Palm Springs, CA. Visionnée sur: https://www.ted.com/talks/ lera_boroditsky_how_language_shapes_the way_we_think?utm_campaign=social\&utm medium $=$ referral\&utm_source $=$ facebook . com\&utm_content=talk\&utm_term =social-science\#t-253678

De Bellefeuille, P. (1993, 29 mars). Féminisation à tous crins: Quand respectera-t-on l'intégrité de la langue française? Le Devoir, p. A7. Récupérée sur http://collections.banq.qc.ca/ ark:/52327/2765332
Dionne-Simard, D., Galarneau, D. \& LaRochelleCôté, S. (2016). Women in scientific occupations in Canada. Statistics Canada Catalogue no. 75-006-X. Ottawa, Canada. Récupérée sur www.statcan.gc.ca/pub/75-006-x/2016001/ article/14643-eng.htm

Evain, A. (2008). Histoire d'autrice, de l'époque latine à nos jours. SÊMÉION: Travaux de sémiologie, 6. Récupérée sur http://siefar. org/wp-content/uploads/2015/09/Histoiredautrice-A_-Evain.pdf

Stoet, G., \& Geary, D. C. (2018). The gender-equality paradox in science, technology, engineering, and mathematics education. Psychological Science, 29, $581-$ 593. doi:10.1177/0956797617741719 